\section{Estudo da umidade de equilibrio de duas espécies de plantas do gênero Phyllanthus após secagem para 0 armazenamento}

\author{
Figueira, G.M ${ }^{1 *}$.; Silva, F. ${ }^{2}$; Magalhães, P.M. ${ }^{\text {; }}$ \\ Park, K.J. ${ }^{2}$
}

${ }^{1}$ Pesquisadores do Centro Pluridisciplinar de Pesquisas Químicas Biológicas e Agrícolas - CPQBA - Unicamp ${ }^{2}$ FEAGRI/UNICAMP

\section{Resumo}

Quebra-Pedra é o nome popular atribuído a espécies do gênero Phyllanthus (Euphorbiaceae). P. niruri e $P$. amarus são estudadas no Brasil e no mundo quanto às suas atividades farmacológicas, decorrentes das indicações populares. O estudo de secagem a armazenamento visa fornecer parâmetros para se obter a matéria prima uniforme e de qualidade. Os objetivos deste trabalho foram determinar o teor de umidade final das espécies e a curva de umidade de equilíbrio (UE) entre 10 e $90 \%$ de UR. As duas espécies foram cultivadas no $\mathrm{CPQBA}$, colhidas a $50 \mathrm{~cm}$ de altura e direcionadas à secagem $\left(\mathrm{T}=50^{\circ} \mathrm{C}\right)$ em estufa com circulação forçada de ar. Na determinação da curva de UE utilizou-se o método estático, empregando-se soluções salinas saturadas. O peso foi controlado através de pesagens periódicas, até atingir o equilíbrio (peso constante), sendo então determinada a UF. Por meio das curvas, determinouse a umidade de equilíbrio característica de cada espécie na faixa de umidade relativa do ar estudada. Os modelos matemáticos que melhor se ajustaram a $P$. amarus foi o de Hasley $(9,3 \%)$, e P.niruri GAB $(8,9 \%)$.
Abstract
Species of the genus Phyllanthus (Euphorbiaceae) is known popularly in Brazil as "Quebra Pedra" (Break-stone) and indicated for the treatment of renal lithiasis. P. niruri and $P$. amarus have been studied in Brazil and around the world regarding pharmacologycal activity, due to folk uses. Drying and storage parameters intend to provide a standard raw material with high quality. The storage condition can determine the durability of raw material and relative humidity is one of the most important factors. The aim of this study was to determine the final percentage of humidity and the desorption isotherms. The species were cultivated on experimental field at CPQBA/UNICAMP. The experimental area was harvest and the aerial part was dried in $50^{\circ} \mathrm{C}$ until constant weight. After dried, the final humidity was determined. The equilibrium moisture had been determined by static gravimetric method, with a range of relative humidity from 10 to $90 \%$. The most commonly used equilibrium moisture content (EMC) and equilibrium relative humidity (ERH) equations were compared with their ability

of best fiting the published sorption data for selected medicinal and aromatic plants. The best-fitted equation identified for P. amarus was Hasley $(9,3 \%)$, and for P.niruri was $\operatorname{GAB}(8,9 \%)$.

O gênero Phyllanthus contém cerca de 600 espécies arbustivas, arbóreas e herbáceas, anuais ou bianuais, distribuídas nas regiões tropicais e subtropicais nos dois hemisférios ${ }^{5}$. A identificação correta dos táxons requer avaliação de especialistas neste gênero, pois em muitos casos, espécies distintas são consideradas variedades. Na década de noventa a reorganização taxonômica do gênero foi realizada, o que contribuiu para diminuir as incorreções de delimitação das espécies importantes, que vêm sendo estudadas quanto aos seus constituintes químicos ${ }^{8}$.

Phyllanthus amarus Schum. \& Thonn e P. niruri L. (Euphorbiaceae) além de outras espécies deste gênero, são conhecidas como "quebra pedra" e são utilizadas popularmente no tratamento de cálculo renal, como diurético e no tratamento de hepatite ${ }^{5}$. À medida que estudos químicos e farmacológicos confirmam cientificamente suas ações terapêuticas, aumenta a demanda por matéria-prima na elaboração destes fitomedicamentos e a busca por produto de alta qualidade torna-se cada vez maior. Sejam estas espécies provenientes do extrativismo ou do cultivo, o material vegetal produzido deve passar pelo processo de secagem e armazenamento a fim de atender às exigências do mercado.

Esta qualidade pode ser preservada através do estudo dos parâmetros de secagem e condições de armazenamento. Informações sobre a umidade de equilíbrio destes materiais tornam-se cada vez mais significantes, pois definem as condições mais adequadas de armazenamento da droga. As isotermas de sorção mostram a relação entre a umidade de equilíbrio do material e a umidade relativa (atividade de água) com temperatura constante, fornecendo as curvas de umidade de equilíbrio ou isotermas de sorção de água. O conhecimento destas curvas é indispensável na determinação do teor de água final necessário na estabilização do produto $^{6}$. A diferença do conteúdo de umidade inicial do material e do conteúdo de umidade de equilíbrio representa a força motriz no processo de secagem. Várias equações empíricas correlacionam os dados experimentais das isotermas de sorção de materiais biológicos. A importância destas equações diz respeito à energia de ligação de água que é traduzida na energia de adsorção. Outra característica é acerca dos valores de monocamada molecular de água, que fornece a superfície disponível na matriz sólida, que indica a relação deste conteúdo com as reações químicas que determinam a deterioração do material.

Os resultados da umidade de equilíbrio são apresentados na tabela 2, assim como os melhores modelos matemáticos constam das figuras 1 e 2, que foram ajustados de acordo com os valores experimentais, considerando os erros abaixo de $10 \%$. 
Tabela 2. Atividade de água e umidade de equilíbrio para as espécies $P$. amarus e $P$. niruri.

\begin{tabular}{l|c|c|c|c}
\hline \multirow{2}{*}{ Sal } & \multicolumn{2}{|c|}{ P. niruri } & \multicolumn{2}{c}{ P. amarus } \\
\cline { 2 - 5 } & \multicolumn{2}{|c|}{$\mathrm{T}=50^{\circ} \mathrm{C}$} & \multicolumn{2}{c}{$\mathrm{T}=50^{\circ} \mathrm{C}$} \\
\hline & $\mathrm{aw}$ & $\mathrm{Xeq}(\mathrm{kg} \mathrm{kg}-1)$ & $\mathrm{Aw}$ & $\mathrm{Xeq}(\mathrm{kg} \mathrm{kg}-1)$ \\
\hline $\mathrm{LiCl}$ & 0.1110 & 0.0768 & 0.1110 & 0.0759 \\
\hline $\mathrm{KF}$ & 0.2080 & - & 0.2080 & - \\
\hline $\mathrm{CaCl} 2$ & 0.2900 & 0.0820 & 0.2900 & 0.0761 \\
\hline $\mathrm{K} 2 \mathrm{CO} 3$ & 0.4000 & 0.0862 & 0.4000 & 0.0871 \\
\hline $\mathrm{NaBr}$ & 0.5093 & 0.0967 & 0.5093 & 0.0992 \\
\hline $\mathrm{KI}$ & 0.6449 & 0.1553 & 0.6449 & 0.1476 \\
\hline $\mathrm{NaCl}$ & 0.7443 & 0.2022 & 0.7443 & 0.1591 \\
\hline $\mathrm{KCl}$ & 0.8120 & 0.2101 & 0.8120 & 0.1651 \\
\hline $\mathrm{KNO} 3$ & 0.8478 & 0.2895 & 0.8478 & - \\
\hline
\end{tabular}

Obs.: valores ausentes na tabela indicam falta de ajuste.

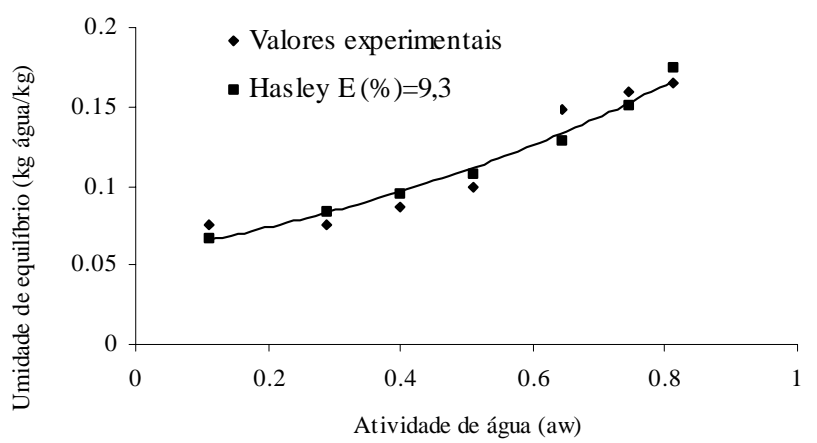

Figura 1. Modelo de Hasley ajustado ao valores experimentais $\left(\mathrm{T}=50^{\circ} \mathrm{C}\right)$ em $P$. amarus.

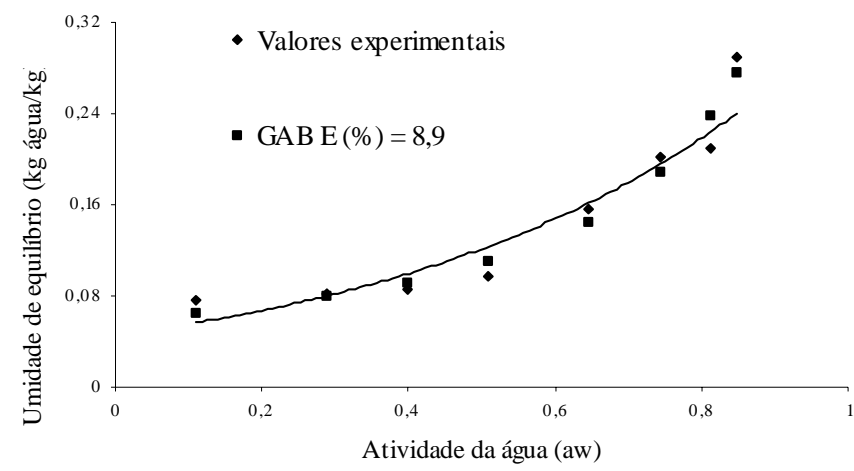

Figura 2. Modelo de GAB ajustado ao valores experimentais $\left(\mathrm{T}=50^{\circ} \mathrm{C}\right)$ em $P$. niruri.

O modelo matemático que melhor se ajustou aos dados experimentais da espécie Pamarus foi Hasley com um erro de $9,3 \%$, e em $P$. niruri foi o modelo de GAB (E = $8,9 \%$ ). Segundo LOMAURO, BASKI, LABUZA ${ }^{4}$, a equação de $G A B$ pode representar com grande precisão mais que $50 \%$ das isotermas de frutas, vegetais e carnes quando comparada com equações de dois parâmetros.

PARK e NOGUEIRA ${ }^{6}$ apresentaram uma conceituação do modelo de BET, chegando até a sua extensão, o modelo de GAB. Concluíram que por apresentar três parâmetros a serem estimados $\left(\mathrm{X}_{\mathrm{m}}, \mathrm{C}_{\mathrm{BET}}, \mathrm{n}\right)$ e sendo a equação polinomial de grau maior que 2 , dependendo do valor numérico de $\mathrm{n}$ estimado, o modelo de BET na sua forma original possivelmente apresentará o melhor ajuste dos dados experimentais.

Vários modelos têm sido investigados no caso das espécies medicinais. De acordo com SILVA et al. (2004), em estudos realizados com Carqueja (Baccharis trimera L.), o modelo que apresentou o menor erro relativo médio foi o de $\mathrm{GAB}$ com $6,76 \%$ na temperatura de $60{ }^{\circ} \mathrm{C}, 7,95 \%$ na temperatura de $30^{\circ} \mathrm{C}$ e $11,95 \%$ no modelo BET Linear à $45^{\circ} \mathrm{C}$.

\section{Material e Métodos}

Plantas das duas espécies foram cultivadas no campo experimental do CPQBA/ UNICAMP em Paulínia, São Paulo. A colheita foi realizada quando as plantas atingiram aproximadamente $50 \mathrm{~cm}$ de altura e o material coletado foi seco em estufa com circulação forçada a $50^{\circ} \mathrm{C}$ até peso constante. Após secagem, foi determinado o teor de umidade final (UF) em estufa a $105 \pm 3^{\circ} \mathrm{C}$ por 24 horas.

Os ensaios de umidade de equilíbrio foram realizados de agosto a dezembro de 2002. Na obtenção da umidade de equilíbrio das espécies estudadas, utilizou-se estufas de circulação de ar forçado, balança digital analítica (precisão de $0,0001 \mathrm{~g}$ ), termômetros, dessecadores, cadinhos de alumínio e soluções salinas.

As amostras com cerca de 2 a 3 gramas, foram colocadas em cadinhos de PVC, em quadruplicatas, dentro de dessecadores cada um contendo uma solução salina saturada diferente simulando nove diferentes condições constantes de umidade relativa (Tabela 1).

Tabela 1. Valores de atividade de água de acordo com a solução salina.

\begin{tabular}{c|c|c|c|c|c|c|c|c|c}
\hline $\mathrm{T}$ & \multicolumn{10}{|c}{ Solução salina } \\
\cline { 2 - 9 }$\left[{ }^{\circ} \mathrm{C}\right]$ & $\mathrm{LiCl}$ & $\mathrm{KF}$ & $\mathrm{CaCl}_{2}$ & $\mathrm{~K}_{2} \mathrm{CO}_{3}$ & $\mathrm{NaBr}$ & $\mathrm{KI}$ & $\mathrm{NaCl}$ & $\mathrm{KCl}$ & $\mathrm{KNO}_{3}$ \\
50 & 11.10 & 20.80 & 35.07 & 40.91 & 50.93 & 64.49 & 74.43 & 81.20 & 84.78 \\
\hline
\end{tabular}

Esses dessecadores foram colocados dentro de estufas de circulação forçada à temperatura de $50^{\circ} \mathrm{C}$ e submetidas ao método gravimétrico padrão (GÁL, 1975), iniciando-se medidas periódicas da redução da massa até tornarem-se equilibradas e constantes. A massa seca foi determinada colocando-se amostras de cada experimento em estufas à $105^{\circ} \mathrm{C}$ por 24 horas (ASAE, $1991 \mathrm{a}$ ).

O método Quasi-Newton (STATISTICA, 1995) de análise de regressão não linear foi usado para estimar as constantes dos modelos. A regressão foi repetida com estimativas de vários valores iniciais acima e abaixo dos calculados (método iterativo: PELEG, 1993). O critério usado na avaliação foi o módulo do erro relativo médio:

$$
E=\frac{100}{n e} \sum_{i=1}^{n e} \frac{|V E-V P|}{V E}
$$

Geralmente, são considerados que valores de erro relativo médio abaixo de $10 \%$ indicam um razoável ajuste 
nas práticas propostas (AGUERRE et al., 1985).

\section{Conclusão}

Através do ajuste das curvas, observou-se que o melhor modelo matemático para P.amarus foi Hasley $(\mathrm{E}=9,3 \%)$ e em $P$. niruri foi o modelo de $\mathrm{GAB}(\mathrm{E}=8,9 \%)$. Dentre estes modelos GAB tem sido um dos mais utilizados para materiais biológicos. A determinação da curva de umidade de equilíbrio nestas espécies pode ser utilizada na determinação do ponto de secagem adequado para manter a qualidade no armazenamento, de modo a preservar sua qualidade intrínseca e a integridade física do produto.

\section{Referências}

${ }^{1}$ Aguerre, R.J.; Gabitto, J.F.; Chirife, J. (1985). Utilization of Fick's second law for the evaluation of diffusion coefficients in food process controlled by internal diffusion. Journal of Food Technology, 20(5), 623-629.

${ }^{2}$ ASAE - American Society of Agricultural Engineers. (1991a). Official Methods of Analysis: D245.4, Moisture Relationship of Grains, 363-367.

${ }^{3}$ Gál, S. (1975). Recent advances in techniques for determination of desorptions isotherms. In: International Symposium, Glasgow: Academic Press Inc, 139-154.

${ }^{4}$ Lomauro, C.J., Bakshi, A.S., Labuza, T.P. (1985). Evaluation of food moisture sorption isotherm equations. Part I: Fruit, vegetable and meat products. Lebensmittel-Wissenschaft \& Technologie, 18(2), 111-117.

${ }^{5}$ Lorenzi, H., Matos, F.J. A. (2002). Plantas Medicinais no Brasil, nativas e exóticas. Instituto Plantarum, Nova Odessa, SP.

${ }^{6}$ Park, K.J., Nogueira, R.I. (1992). Modelos para ajuste de isotermas de sorção de alimentos, Engenharia Rural, 3(1), 180-186.

${ }^{7}$ Peleg, M. (1993). Assessment of a semi-empirical four parameter general model for sigmoid moisture sorption isotherms. J. of Food Processing Engineering, 16(1), 21-37.

${ }^{8}$ Raintree (2004). <http://rain-tree.com/>, 15/08/2004.

${ }^{9}$ Silva, F.; Park, K. J. ; Magalhães, P.M.; Pozitano, M. (2004). Estudo de secagem de carqueja (Baccharis genistelloides L.) Anais do XXXIII Congresso Brasileiro de Engenharia Agricola , CD-ROM.

${ }^{10}$ Statistica for Windows 5.0. (1995). Computer program manual. StatSoft, Inc., Tulsa.

\section{Autora para correspondência}

Glyn Mara Figueira

Centro Pluridisciplinar de Pesquisas Químicas

Biológicas e Agrícolas - CPQBA - Unicamp

Caixa Postal 6171, 13081-970 - Campinas (SP)

e-mail: glyn@cpqba. unicamp.br 